\title{
Geometry dependence of Auger carrier capture rates into cone-shaped self- assembled quantum dots
}

Magnúsdóttir, Ingibjörg; Bischoff, Svend; Uskov, A.V.; Mørk, Jesper

Published in:

Physical Review B Condensed Matter

Link to article, DOI:

10.1103/PhysRevB.67.205326

Publication date:

2003

Document Version

Publisher's PDF, also known as Version of record

Link back to DTU Orbit

Citation (APA):

Magnúsdóttir, I., Bischoff, S., Uskov, A. V., \& Mørk, J. (2003). Geometry dependence of Auger carrier capture rates into cone-shaped self-assembled quantum dots. Physical Review B Condensed Matter, 67(20), 205326. https://doi.org/10.1103/PhysRevB.67.205326

\section{General rights}

Copyright and moral rights for the publications made accessible in the public portal are retained by the authors and/or other copyright owners and it is a condition of accessing publications that users recognise and abide by the legal requirements associated with these rights.

- Users may download and print one copy of any publication from the public portal for the purpose of private study or research.

- You may not further distribute the material or use it for any profit-making activity or commercial gain

- You may freely distribute the URL identifying the publication in the public portal 


\title{
Geometry dependence of Auger carrier capture rates into cone-shaped self-assembled quantum dots
}

\author{
I. Magnusdottir, S. Bischoff, A. V. Uskov,* and J. Mørk \\ COM, Technical University of Denmark, Bldg. 345w, DK-2800 Kgs. Lyngby, Denmark
}

(Received 11 December 2002; published 30 May 2003)

\begin{abstract}
We calculate carrier capture rates into cone- and truncated-cone-shaped quantum dots mediated by Auger processes. It is demonstrated that the capture rates depend strongly on both dot size and shape. The importance of phonon-mediated versus the Auger-mediated capture processes is discussed.
\end{abstract}

DOI: 10.1103/PhysRevB.67.205326

PACS number(s): 73.21.La

\section{INTRODUCTION}

The electrical and optical properties of quantum-dot (QD) structures have been studied intensely in recent years. The growth technology for such structures has matured very rapidly, leading to the realization of lasers based on selfassembled QD's with record low-threshold current densitites ${ }^{1}$ and high output power. ${ }^{2,3}$ QD based optical amplifiers may also have a number of interesting properties. ${ }^{4,5}$ Carriers in such devices are pumped electrically to layers around the dots. They are then captured into the discrete levels of the dots, where they relax to the lower-lying levels and recombine radiatively. These capture and relaxation processes have been studied both experimentally and theoretically, ${ }^{6-14}$ and have been considered to be mediated by Auger processes (carrier-carrier interaction) ${ }^{6,9,10,13}$ and carrier-phonon interaction. ${ }^{11,12,14}$ In time-resolved photoluminescence (PL) experiments, carrier capture times (PL rise times) are typically measured to be in the range of a few to several tens of ps. ${ }^{715-17}$ Relaxation by emission of longitudinal optical (LO) phonons is an efficient mechanism in semiconductors of higher dimensionality. For phononmediated capture into QD's, the range of dot sizes, where such capture processes are allowed, is limited due to the nearly dispersionless LO phonons and the requirement that energy is conserved in the process. Carrier capture times by emission of one LO phonon have been calculated to be a few ps at resonance for carrier sheet density $n=10^{15} \mathrm{~m}^{-2}{ }^{11}$ Two-phonon mediated capture times have been shown to be about an order of magnitude larger. ${ }^{14}$

Auger carrier capture rates have been calculated for capture into a box-shaped dot embedded in a quantum well $(\mathrm{QW}),{ }^{6}$ a spherical $\operatorname{dot}^{8}{ }^{8}$ a truncated cone, ${ }^{9}$ and a cylindershaped dot. ${ }^{10}$ In this paper we report calculations of Auger capture rates into InAs/GaAs cone-shaped self-assembled QD's and study in detail the capture-rate dependence on the QD geometry. In particular, we identify a strong dependence of the capture rates on the level separation between the wetting-layer (WL) band edge and the dot level to which the capture takes place.

Two types of Auger capture processes have been suggested. One type of process, labeled type $\mathrm{I},{ }^{10}$ is one where a WL electron or hole interacts with another WL electron or hole, resulting in a carrier captured by the QD while the other is scattered to a higher energy in the WL. ${ }^{6,9,10}$ Two examples of such a process are illustrated in Fig. 1. The left panel shows capture of an electron by scattering with another electron. The right panel shows capture of a hole by scattering with an electron. In another type of process (type II) a WL electron (hole) is captured by the QD while a QD hole (electron) is excited out of the dot into the WL. The capture process of type II has been shown to yield capture times close to $1 \mathrm{ps}$ (counting spin degeneracy) at carrier densities $\sim 10^{15} \mathrm{~m}^{-2}$ but only for a small interval of dot sizes. ${ }^{10}$ In the following we consider the Auger carrier capture process of type I.

\section{MODEL}

The carrier capture rate, i.e., the rate of carriers making a transition from the WL to a QD state, can be determined by Fermi's golden rule,

$$
\begin{aligned}
R= & \frac{2 \pi}{\hbar} \sum_{\text {spin config }} \sum_{\mathbf{k}_{s}} \sum_{\mathbf{k}_{s}^{\prime}} \sum_{\mathbf{k}_{c}}\left|\left\langle d, \mathbf{k}_{s}^{\prime}\left|V_{c}\right| \mathbf{k}_{c}, \mathbf{k}_{s}\right\rangle\right|^{2} f\left(\boldsymbol{\epsilon}_{k_{s}}\right) f\left(\boldsymbol{\epsilon}_{k_{c}}\right) \\
& \times\left[1-f\left(\epsilon_{k_{s}^{\prime}}\right)\right] \delta\left(E_{f}-E_{i}\right),
\end{aligned}
$$

where $V_{c}$ is the Coulomb interaction. $\mathbf{k}_{c}$ and $\mathbf{k}_{s}$ are wave vectors characterizing the captured and scattered carrier initial states, $\mathbf{k}_{s}^{\prime}$ denotes the final state of the scattered carrier, and $|d\rangle$ is the (final) quantum-dot state. The sign $\Sigma_{\text {spin config }}$ in Eq. (1) is a sum over all possible spin configurations of two interacting particles. $E_{i}\left(E_{f}\right)$ is the energy of the initial (final) state.

For carrier sheet densities in the WL ranging up to values of $n \sim 10^{16} \mathrm{~m}^{-2}$, the carrier capture rate can be written as $R=C n^{2}$, where $C$ is a proportionality constant. This is be-

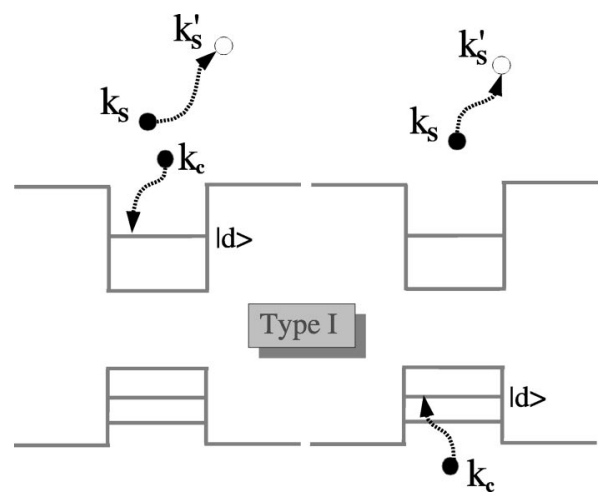

FIG. 1. Auger capture processes of type I. 

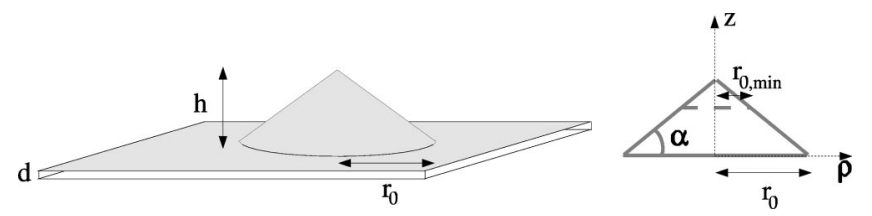

FIG. 2. Cone-shaped quantum dot situated on a wetting layer of thickness $d$. $h$ is the cone height, $r_{0}$ the in-plane base radius and $\alpha$ is the base angle. The cone can also be truncated, shown in the right panel with a dashed line. The upper radius is labeled by $r_{0, \min }$ and the height is then determined by $h=\tan \alpha\left(r_{0}-r_{0, \min }\right)$.

cause the Fermi factors $f\left(\epsilon_{k_{s}}\right)$ and $f\left(\epsilon_{k_{c}}\right)$ in Eq. (1) can be approximated by a Boltzmann factor which, in turn, is proportional to $n$. We can, to a good approximation, write the factor $\left[1-f\left(\epsilon_{k_{s}^{\prime}}\right)\right] \approx 1$. This Auger coefficient ${ }^{10}$ will be labeled $C_{\mathrm{cs}}$, where the indices indicate the type of the captured and scattered carrier, respectively, with $c=e, h$ and $s=e, h$. Note that the Coulomb matrix element in Eq. (1) involves a sum over all possible spin states. In the case where the two particles are identical, an antisymetrization of the wave functions results in direct and exchange Coulomb integrals. We neglect the interference term between direct and exchange Coulomb matrix elements. The direct and exchange terms contribute equally to the total capture rate.

We model the QD's by truncated or nontruncated cones with a finite confinement potential. The QD geometry is illustrated in Fig. 2. The left panel shows a nontruncated cone situated on a WL of thickness $d$. The right panel shows the dot parameters that are used for a truncated or a nontruncated cone.

In order to calculate the eigenenergies and obtain wave functions of a relatively simple form, that can be used in subsequent calculations, we use variational wave functions of the type presented in Ref. 11 in the effective-mass approximation. The states are characterized by the quantum numbers $\ell$ and $m$, where $\ell-1$ is the number of radial nodes and $m$ is an angular momentum quantum number that describes rotation around the $z$-axis. We label states by $|\ell S\rangle$ $(m=0),|\ell P\rangle(m= \pm 1),|\ell D\rangle(m= \pm 2), \ldots$ The QD height is denoted by $h$, the in-plane base radius by $r_{0}$ and the base angle by $\alpha$. In the following we will present results for capture into the lowest-lying energy states $|1 S\rangle$ and $|1 P\rangle$.

The QD wave functions are approximated by

$$
\Psi_{d}(\mathbf{r})=\Phi_{d}(\boldsymbol{\rho}) \zeta_{d}(z),
$$

i.e., by the product of an envelope function $\zeta_{d}$, in the growth direction $z$, and an envelope function $\Phi_{d}$ that depends on the coordinates in the plane of the WL. We also approximate the $\mathrm{WL}$ wave functions in the same manner,

$$
\Psi_{k}(\mathbf{r})=\Phi_{k}(\boldsymbol{\rho}) \zeta_{w}(z),
$$

where $\Phi_{k}$ is a plane wave and $\zeta_{w}$ is the $z$-dependent part of the WL wave function that is approximated by the solution to a thin QW with the same confinement potential as the QD. In the following we calculate the direct Coulomb matrix element. We have that

$$
\begin{aligned}
& \left\langle d, \mathbf{k}_{s}^{\prime}\left|V_{c}\right| \mathbf{k}_{c}, \mathbf{k}_{s}\right\rangle \\
& =\int d^{2} \rho_{1} d^{2} \rho_{2} \Phi_{d}^{*}\left(\boldsymbol{\rho}_{1}\right) \Phi_{k_{s}^{\prime}}^{*}\left(\boldsymbol{\rho}_{2}\right) \Phi_{k_{c}}\left(\boldsymbol{\rho}_{1}\right) \Phi_{k_{s}}\left(\boldsymbol{\rho}_{2}\right) \\
& \quad \times \int d z_{1} d z_{2} \zeta_{d}^{*}\left(z_{1}\right) \zeta_{w}^{*}\left(z_{2}\right) V_{c}\left(\left|\mathbf{r}_{1}-\mathbf{r}_{2}\right|\right) \zeta_{w}\left(z_{1}\right) \zeta_{w}\left(z_{2}\right) .
\end{aligned}
$$

Due to the thin WL, the $z$-dependent part of the WL wave function is assumed to the described by the ground state of a one-dimensional QW. We can express the Coulomb interaction in terms of a two-dimensional (2D) Fourier transform as

$$
\begin{aligned}
V_{c}\left(\left|\mathbf{r}_{1}-\mathbf{r}_{2}\right|\right) & =\frac{e_{c} e_{s}}{4 \pi \epsilon_{r} \epsilon_{0}\left|\mathbf{r}_{1}-\mathbf{r}_{2}\right|} \\
& =\frac{e_{c} e_{s}}{2 \epsilon_{r} \epsilon_{0}} \frac{1}{S} \sum_{\boldsymbol{\beta}_{\perp}} \frac{e^{-i \boldsymbol{\beta}_{\perp} \cdot\left(\boldsymbol{\rho}_{1}-\boldsymbol{\rho}_{2}\right)}}{\beta_{\perp}} e^{-\beta_{\perp}\left|z_{1}-z_{2}\right|},
\end{aligned}
$$

where $e_{c}$ and $e_{s}$ are the charges of the captured and scattered carrier, respectively, $\epsilon_{r}$ is the dielectric constant and $\epsilon_{0}$ is the permittivity. $\boldsymbol{\beta}_{\perp}$ is a $2 \mathrm{D}$ wave vector in the plane of the $\mathrm{WL}$ and the normalization area of the WL is denoted by $S$. We should mention that we have used the simplifying approximation of an unscreened Coulomb potential. This transform allows us to write the Coulomb matrix element from Eq. (4) as

$$
\begin{aligned}
& \left\langle d, \mathbf{k}_{s}^{\prime}\left|V_{c}\right| \mathbf{k}_{s}, \mathbf{k}_{c}\right\rangle \\
& =\frac{e^{2}}{2 \epsilon_{r} \epsilon_{0}} \frac{1}{S} \sum_{\boldsymbol{\beta}_{\perp}} \frac{1}{\beta_{\perp}} F\left(\beta_{\perp}\right) \\
& \quad \times \int d^{2} \rho_{1} d^{2} \rho_{2} e^{-i \boldsymbol{\beta}_{\perp} \cdot\left(\boldsymbol{\rho}_{1}-\boldsymbol{\rho}_{2}\right)} \Phi_{k_{s}^{\prime}}^{*}\left(\boldsymbol{\rho}_{2}\right) \Phi_{d}^{*}\left(\boldsymbol{\rho}_{1}\right) \\
& \quad \times \Phi_{k_{s}}\left(\boldsymbol{\rho}_{2}\right) \Phi_{k_{c}}\left(\boldsymbol{\rho}_{1}\right),
\end{aligned}
$$

where $F\left(\beta_{\perp}\right)$ is the form factor in which the $z$ part of the wave functions is contained,

$$
F\left(\beta_{\perp}\right)=\iint d z_{1} d z_{2}\left|\zeta_{w}\left(z_{2}\right)\right|^{2} e^{-\beta_{\perp}\left|z_{1}-z_{2}\right|} \zeta_{w}\left(z_{1}\right) \zeta_{d}^{*}\left(z_{1}\right)
$$

As mentioned earlier, we model the in-plane part of the WL wave functions by plane waves. The integration over $\boldsymbol{\rho}_{2}$ thus gives

$$
\int d^{2} \rho_{2} e^{i \boldsymbol{\beta}_{\perp} \cdot \boldsymbol{\rho}_{2}} \Phi_{k_{s}^{\prime}}^{*}\left(\boldsymbol{\rho}_{2}\right) \Phi_{k_{s}}(\boldsymbol{\rho})=\delta_{\boldsymbol{\beta}_{\perp}, \mathbf{k}_{s}^{\prime}-\mathbf{k}_{s}},
$$

which describes conservation of crystal momentum in the WL continuum. We can therefore simplify the Coulomb matrix element further into 


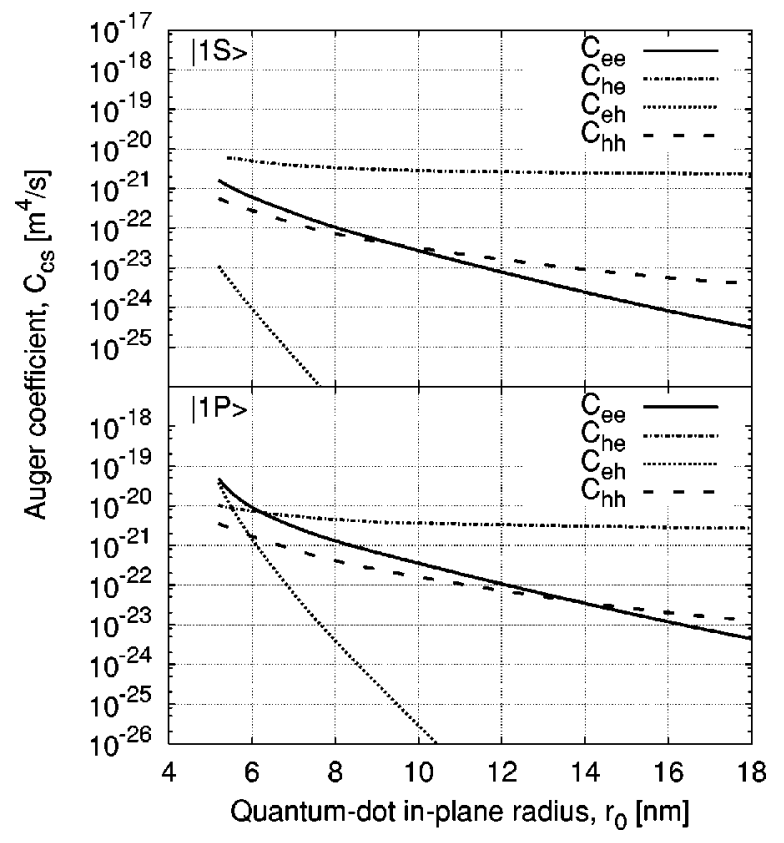

FIG. 3. The Auger capture coefficients $C_{\text {ee }}, C_{\text {he }}, C_{\text {eh }}, C_{\text {hh }}$ as a function of quantum dot in-plane radius for carrier capture into the state $|1 S\rangle$ (upper panel) and $|1 P\rangle$ (lower panel).

$$
\begin{aligned}
\left\langle d, \mathbf{k}_{s}^{\prime}\left|V_{c}\right| \mathbf{k}_{s}, \mathbf{k}_{c}\right\rangle= & \frac{e^{2}}{2 \epsilon_{r} \epsilon_{0}} \frac{1}{S} \frac{F\left(\mathbf{k}_{s}^{\prime}-\mathbf{k}_{s}\right)}{\left|\mathbf{k}_{s}^{\prime}-\mathbf{k}_{s}\right|} \\
& \times \int d^{2} \rho e^{i\left(\mathbf{k}_{s}-\mathbf{k}_{s}^{\prime}\right) \cdot \boldsymbol{\rho}^{*}}(\boldsymbol{\rho}) \Phi_{k_{c}}(\boldsymbol{\rho}) .
\end{aligned}
$$

Because $\Phi_{k_{c}}(\boldsymbol{\rho})$ is a plane wave, the remaining integral is essentially a Fourier transform of the QD wave function with respect to $\left(\mathbf{k}_{s}-\mathbf{k}_{s}^{\prime}+\mathbf{k}_{c}\right)$.

\section{RESULTS}

Figure 3 shows the calculated Auger coefficients as a function of quantum-dot in-plane radius for carrier capture into $|1 S\rangle$ and $|1 P\rangle$, respectively. We have set $h=3 \mathrm{~nm}, \alpha$ $=30^{\circ}, d=0.33 \mathrm{~nm}, \epsilon_{r}=12.5$, and $T=300 \mathrm{~K}$. The confinement potential is set to be $V_{e}=697 \mathrm{meV}$ and $V_{h}$ $=288 \mathrm{meV}$ for electrons and holes, respectively. The effective masses are set to be $m_{e}^{*}=0.07 m_{0}$ and $m_{h}^{*}=0.34 m_{0}$. The results in Fig. 3 show that $C_{\mathrm{ee}}>C_{\mathrm{eh}}$ and $C_{\mathrm{he}}>C_{\mathrm{hh}}$, i.e., for capture of a given type of carrier, scattering by electrons is in general more effective than scattering by holes. The same trends are seen if the coefficients are plotted as a function of the energy spacing between the wetting-layer band edge and the quantum-dot energy level that the carrier is captured into. This is shown in Fig. 4 for capture of electrons and in Fig. 5 for capture of holes, where we have set $\Delta E=E_{\mathrm{WL}}-E_{d}$, where $E_{\mathrm{WL}}$ is the energy of the WL band edge and $E_{d}$ is the energy of the QD state. The more efficient scattering by electrons can be explained by assuming a given energy spacing between the band edge and the quantum-dot level. For an incident carrier of given energy $\epsilon_{k_{c}}$, the energy that is trans-

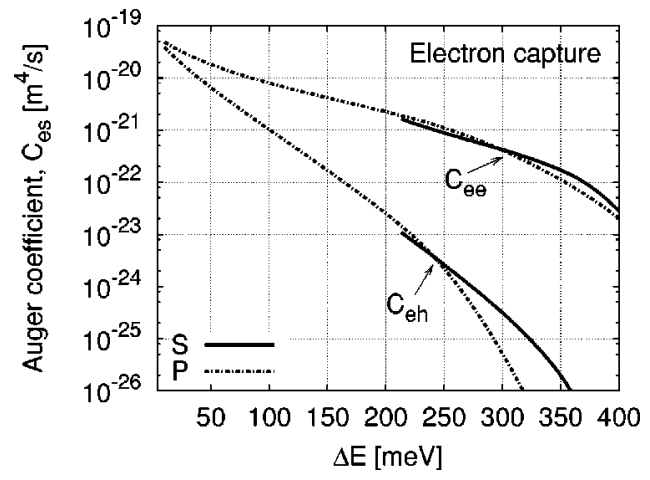

FIG. 4. The Auger capture coefficients for capture of electrons, $C_{\text {ee }}$ and $C_{\text {eh }}$, as a function of the energy separation between the wetting-layer band edge and the energy level that the carrier is captured into.

ferred to the scattered carrier, that is either an electron or hole, is the same due to the energy conservation requirement. However, due to the smaller curvature in the energy dispersion for the holes the mean "momentum transfer" so to say, $\left\langle\left|\mathbf{k}_{s}^{\prime}-\mathbf{k}_{s}\right|\right\rangle$, is in general larger for holes. The Coulomb matrix element decreases with increasing $\left|\mathbf{k}_{s}^{\prime}-\mathbf{k}_{s}\right|$, mainly because the $1 /\left|\mathbf{r}_{1}-\mathbf{r}_{2}\right|$ dependence of the Coulomb interaction translates into a $1 /\left|\mathbf{k}_{s}^{\prime}-\mathbf{k}_{s}\right|$ dependence in $k$ space. Furthermore, the form factor $F\left(\mathbf{k}_{s}^{\prime}-\mathbf{k}_{s}\right)$ decreases with increasing $\left(\mathbf{k}_{s}^{\prime}-\mathbf{k}_{s}\right)$. Hence scattering by holes is in general less efficient than scattering by electrons.

It is clear from Figs. 4 and 5 that for a given process the capture rate depends mainly on the energy separation $\Delta E$ and not so much on the type of QD state that the carrier is captured into. This can be understood in terms of the wave functions. Thus, for a given energy separation, the characteristic decay constants are similar and hence the wave function overlaps that enter the capture rate are similar. We can go even further in our comparison by considering capture processes, where the scattered carrier is of the same type but the captured ones are different. If we look at $C_{\text {ee }}$ and $C_{\text {he }}$ in the lower panel of Fig. 3 for capture into $|1 P\rangle$ we see that $C_{\text {he }}$ crosses $C_{\mathrm{ee}}$ at $r_{0} \approx 6.5 \mathrm{~nm}\left(C_{\mathrm{he}}=C_{\mathrm{ee}} \approx 8 \times 10^{-21} \mathrm{~m}^{4} / \mathrm{s}\right)$ and is larger than $C_{\mathrm{ee}}$ beyond this radius. An inspection of Figs. 4

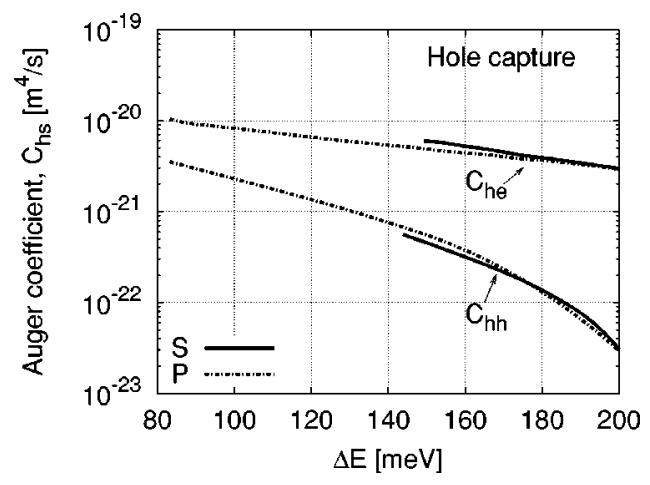

FIG. 5. The Auger capture coefficients for capture of holes, $C_{\text {he }}$ and $C_{\mathrm{hh}}$, as a function of the energy separation between the wetting-layer band edge and the energy level that the carrier is captured into. 


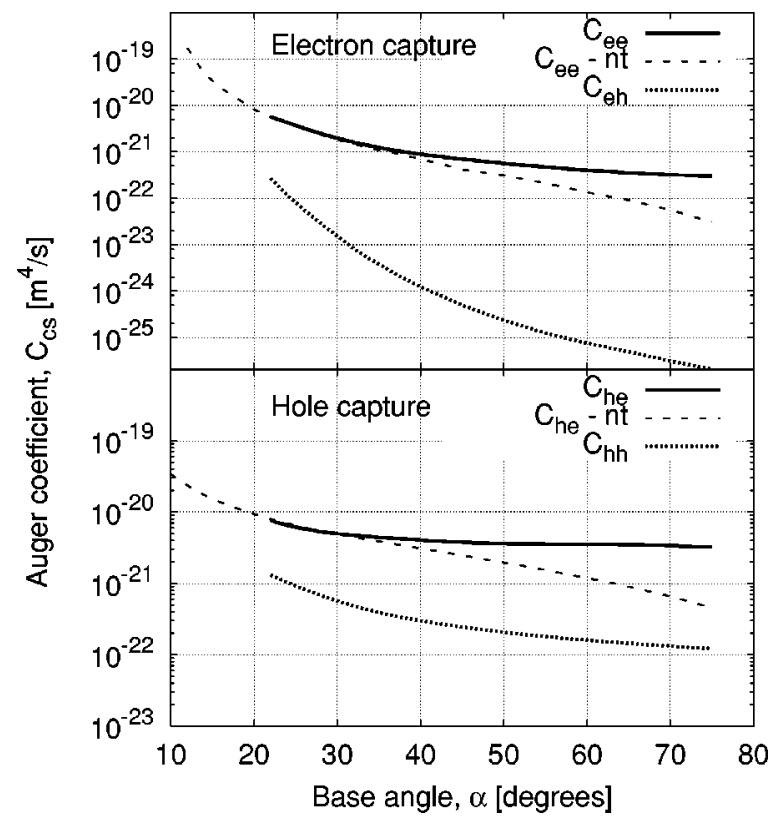

FIG. 6. The Auger coefficients $C_{\text {ee }}$ and $C_{\text {he }}$ plotted versus the base angle for a truncated and nontruncated ("nt") cone with inplane radius $r_{0}=7.5 \mathrm{~nm}$. The height of the truncated cone is set to be $h=3 \mathrm{~nm}$.

and 5 shows that this crossing point occurs at approximately the same energy level separation $\Delta E \sim 100 \mathrm{meV}$. The slower decrease of $C_{\text {he }}$ compared to $C_{\text {ee }}$ in Fig. 3 hence simply reflects the fact that the energy separation from the WL increases more slowly with increasing $r_{0}$ for the hole levels than for the electron levels. The results in Figs. 4 and 5 also explain why the coefficients found here decrease much faster with dot size than in Ref. 10, where the energy spacing between the WL band edge and the QD level is held constant. The oscillations of $C_{\mathrm{cs}}$ with dot size, that were found in Ref. 10 for capture into the ground state of a cylinder-shaped dot, but are absent here, arise because the in-plane wave functions are approximated by solutions to the Schrödinger equation with an infinite confinement potential.

We show in Fig. 6 the base-angle dependence of $C_{\mathrm{cs}}$ for capture into $|1 P\rangle$ for a "regular" cone and a truncated cone. The base radius is constant, $r_{0}=7.5 \mathrm{~nm}$, and the height of the truncated cone is $h=3 \mathrm{~nm}$. The upper panel shows capture of electrons and the lower panel capture of holes. Increasing $\alpha$ corresponds to approaching a cylinder-shaped dot ( $\alpha$ $=90^{\circ}$ ) and increasing the dot volume. This means that the states become more deeply bound to the dot which leads to decreasing coefficients. We show results up to $\alpha=75^{\circ}$. No variational solution can be found beyond $\alpha=75^{\circ}$ with the trial functions that we use here. At larger $\alpha$, the coefficients $C_{\text {ee }}$ and $C_{\text {he }}$ for capture into a nontruncated cone decrease faster with $\alpha$ than the corresponding coefficients for capture into a truncated cone. This arises mainly because the wave function for a nontruncated cone is localized farther away from the WL (it is more deeply bound) than for the truncated cone, and this gives lower overlaps of QD and WL wave functions.

The capture rate of an electron, by scattering with an elec- tron, into the first excited state of a quantum box of base length $50 \mathrm{~nm}$, has been found to be $R=2 \times 10^{11} \mathrm{~s}^{-1}$ at $n$ $=10^{15} \mathrm{~m}^{-2},{ }^{6}$ which gives $C_{\mathrm{ee}}=5 \times 10^{-20} \mathrm{~m}^{4} / \mathrm{s}$. This coefficient could be compared to our results if we extrapolate $C_{\text {ee }}$ in Fig. 6 to $\alpha=90^{\circ}$. The results obtained in Ref. 6 give much higher capture rates. This may be due to a large overlap of QW and QD wave functions because the QD is embedded in the QW. In contrast, our approach assumes WL carriers with in-plane wave-function components that are unaffected by the dots. In reality, the wave functions of continuum carriers will be perturbed in the proximity of dots, which may eventually lead to quasibound states that may alter the scattering rate. $^{18}$

If the coefficients of Fig. 6 are plotted versus the energy separation $\Delta E$ we obtain coefficients very similar to those obtained in Figs. 4 and 5. In particular, capture into a truncated or nontruncated cone for a given process $C_{\mathrm{cs}}$ depends solely on $\Delta E$. This demonstrates that the dependence of $C_{\mathrm{cs}}$ on $\Delta E$ is strong and our investigations indicate that the correspondence is nearly one to one for a given type of process, at least for the confinement potential and particle masses that we have used here, and within the approximation of the variational wave functions that we have used.

We have also computed phonon-mediated capture by emission of one longitudinal optical (LO) phonon for a truncated cone. Details of the model may be found in Ref. 11. Due to the energy conservation requirement, the energy of the QD level that is captured into must fulfill $E_{d}>E_{\mathrm{WL}}$ $-\hbar \omega_{\mathrm{LO}}$, where $\hbar \omega_{\mathrm{LO}}$ is the LO phonon energy. We assume here that the LO phonons are dispersionless. In strongly confined self-assembled QD's, the energy of the QD states decreases quickly with increasing dot size. Due to this and the stringent energy conservation requirement, single-phonon capture processes (emission of one LO phonon) are only allowed for a small range of dot sizes. However, at resonance, they are very efficient. The capture time (inverse of the capture rate) has been shown to be $\sim 1$ ps at $n=10^{15} \mathrm{~m}^{-2}$ for one-phonon processes at room temperature. Two-phonon processes have been shown to give slightly longer capture times. ${ }^{14}$ For two-phonon processes, the energy of the QD level that is captured into, must fulfill $E_{d}>E_{\mathrm{WL}}-2 \hbar \omega_{\mathrm{LO}}$ $\left(\Delta E<2 \hbar \omega_{\mathrm{LO}}\right)$, i.e., capture is allowed into levels that are lying too deep to be reached in a single-phonon process. Hence the dot size interval, for which the two-phonon capture process is allowed, is larger than for single-phonon capture. At resonance for single-phonon electron capture, we plot the Auger carrier capture rates and single-phonon capture rates in Fig. 7 as a function of carrier sheet density. For the dot geometry that we study here, the hole level is too deeply bound to be reached in a single-phonon process. We therefore only show electron phonon capture. At low to moderate carrier densities, phonon capture is proportional to $n$ because only one carrier is involved in the capture process. In contrast, Auger capture of the type that we investigate here, is proportional to $n^{2}$ because two WL carriers are involved in the process. It is clear from Fig. 7 that phononmediated capture processes dominate over Auger processes for densities ranging up to $\sim 5 \times 10^{16} \mathrm{~m}^{-2}$. Again, however, it has to be emphasized that phonon-mediated capture is only 


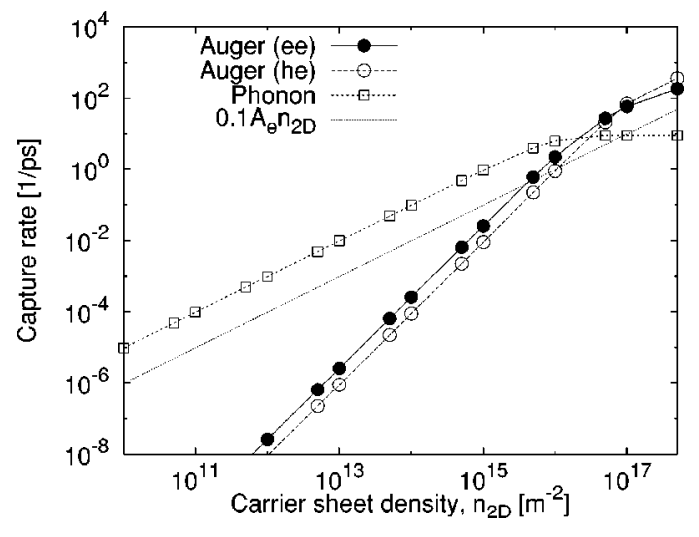

FIG. 7. Auger carrier capture rates of electrons and holes by scattering of electrons compared to capture of electrons with emission of one LO phonon. We have set $r_{0}=5.4 \mathrm{~nm}, h=3 \mathrm{~nm}$ and $\alpha$ $=30^{\circ}$. The Auger coefficients are found to be $C_{\mathrm{ee}}=5.2$ $\times 10^{-20} \mathrm{~m}^{4} / \mathrm{s}$ and $C_{\mathrm{he}}=9.0 \times 10^{-21} \mathrm{~m}^{4} / \mathrm{s}$ and the phonon-assisted capture constant to be $A_{e}=1.0 \times 10^{-3} \mathrm{~m}^{2} / \mathrm{s}$. The dotted line indicates the approximate level of two-phonon capture.

effective in narrow ranges of QD radii. Two-phonon capture rates have been shown to be about one order of magnitude smaller than single-phonon capture rates. ${ }^{14}$ Thus the rate $R$ $=0.1 A_{e} n$, where $A_{e}$ is the phonon-assisted capture constant, is also plotted in Fig. 7 to illustrate the order-of-magnitude estimate for the two-phonon capture rate. We see that Auger capture rates only exceed the two-phonon-assisted capture rate at $n \approx 10^{16} \mathrm{~m}^{-2}$. In view of the trends in the capture rates when going from single-phonon to two-phonon emission, capture rates by multiphonon emission are expected to be lowered with increasing number of emitted phonons. At the same time the size range, where these multiphonon processes are possible, increases. The energy level spacing $\Delta E$ can in many cases be rather large, maybe on the order of 100 $\mathrm{meV}$. Therefore such multiphonon processes can play an important role in the capture mechanism into dots. The efficiency of Coulomb- versus phonon-mediated capture hence depends largely on the energy level spacing between the WL band edge and the higher-lying dot levels, which, in selfassembled QD structures, varies due to the inhomogeneous broadening. Such processes may therefore be competing due to the variation in $\Delta E$ from dot to dot.

In conclusion, we have calculated Auger carrier capture rates to the QD ground state and the first excited state. We have shown that the capture rates are larger for smaller dot sizes and that they decrease very quickly with the dot size. We have shown that Auger capture rates depend very strongly on $\Delta E$, the energy level separation between the WL band edge and the QD level into which capture occurs. For the confinement potential and effective masses that we have used here, the Auger coefficients were shown to depend solely on $\Delta E$ when in-plane QD radius or base angle were varied. Capture by scattering of electrons is in general more efficient than scattering by holes. The largest coefficients are in the range $C_{\mathrm{cs}} \approx 5 \times 10^{-20} \mathrm{~m}^{4} / \mathrm{s}$, which corresponds to a capture rate of about $5 \times 10^{11} \mathrm{~s}^{-1}$ (capture time $2 \mathrm{ps)}$ ) at $n$ $=3 \times 10^{15} \mathrm{~m}^{-2}$. We used the same model for the QD wave functions to calculate phonon-assisted capture rates and compared them to Auger capture rates. Single-phonon capture rates were shown to be dominant over Auger capture rates when single-phonon processes are energetically allowed. However, due to the relatively large variation in $\Delta E$ due to the inhomogeneous broadening in self-assembled dots, we suggested that multiphonon- and Coulomb-mediated processes both contribute to capture.

\section{ACKNOWLEDGMENTS}

The work of one of the authors (A.V.U.) was supported by the Russian Federal Program Integration (Project No. A0155), by RFBR (Project No. 01-02-17330), by INTAS (Project No. 2001-0571), by the Danish Research Council within the framework of program SCOOP, and by the Otto Moensted Foundation. R. Ferreira is gratefully acknowledged for supplying the program code used for calculating the variational wave functions. Bjarne Tromborg is gratefully acknowledged for fruitful discussions.
*Also at Lebedev Physical Institute, Leninsky Prospect 53, 117924 Moscow, Russia.

${ }^{1}$ M. Grundmann, Physica E 5, 167 (2000).

${ }^{2}$ M. Grundmann, F. Heinrichsdorff, N. N. Ledentsov, Ch. Ribbat, D. Bimberg, A. E. Zhukov, A. R. Kovsh, M. V. Maximov, Y. M. Shernyakov, D. A. Lifshits, V. M. Ustinov, and Zh. I. Alferov, Jpn. J. Appl. Phys., Part 1 39, 2341 (2000).

${ }^{3}$ J. Reithmaier, F. Klopf, and R. Krebs, in 2001 IEEE/LEOS Annual Meeting Conference Proceedings (IEEE, San Diego, 2001), Vol. 1, p. 269.

${ }^{4}$ P. Borri, W. Langbein, J. M. Hvam, F. Heinrichsdorff, M.-H. Mao, and D. Bimberg, IEEE Photonics Technol. Lett. 12, 594 (2000).

${ }^{5}$ T. W. Berg, S. Bischoff, I. Magnusdottir, and J. Mørk, IEEE Photonics Technol. Lett. 13, 541 (2001).

${ }^{6}$ U. Bockelmann and T. Egeler, Phys. Rev. B 46, 15574 (1992).

${ }^{7}$ B. Ohnesorge, M. Albrecht, J. Oshinowo, A. Forchel, and Y. Arakawa, Phys. Rev. B 54, 11532 (1996).

${ }^{8}$ J. L. Pan, Phys. Rev. B 49, 11272 (1994).
${ }^{9}$ M. Braskén, M. Lindberg, and J. Tulkki, Phys. Status Solidi A 164, 427 (1997).

${ }^{10}$ A. V. Uskov, J. McInerney, F. Adler, and H. Schweizer, Appl. Phys. Lett. 72, 58 (1998).

${ }^{11}$ R. Ferreira and G. Bastard, Appl. Phys. Lett. 74, 2818 (1999).

${ }^{12}$ T. Inoshita and H. Sakaki, Phys. Rev. B 56, R4355 (1997).

${ }^{13}$ S. Raymond, K. Hinzer, S. Fafard, and J. L. Merz, Phys. Rev. B 61, R16 331 (2000).

${ }^{14}$ I. Magnusdottir, A. V. Uskov, S. Bischoff, B. Tromborg, and J. Mørk, J. Appl. Phys. 92, 5982 (2002).

${ }^{15}$ S. Marcinkevicius and R. Leon, Phys. Rev. B 59, 4630 (1999).

${ }^{16}$ M. De Giorgi, C. Lingk, G. von Plessen, J. Feldmann, S. De Rinaldis, A. Passaseo, M. De Vittorio, R. Cingolani, and M. Lomascolo, Appl. Phys. Lett. 79, 3968 (2001).

${ }^{17}$ T. S. Sosnowski, T. B. Norris, H. Jiang, J. Singh, K. Kamath, and P. Bhattacharya, Phys. Rev. B 57, R9423 (1998).

${ }^{18}$ I. Magnusdottir, A. V. Uskov, R. Ferreira, G. Bastard, J. Mørk, and B. Tromborg, Appl. Phys. Lett. 81, 4318 (2002). 